\title{
Finite-Temperature Quasicontinuum: Molecular Dynamics without All the Atoms
}

\author{
L. M. Dupuy, ${ }^{1}$ E. B. Tadmor, ${ }^{2}$ R. E. Miller, ${ }^{3}$ and R. Phillips ${ }^{4}$ \\ ${ }^{1}$ Lawrence Livermore National Laboratory, L-415, Livermore, California 94551, USA \\ ${ }^{2}$ Faculty of Mechanical Engineering, Technion - Israel Institute of Technology, 32000 Haifa, Israel \\ ${ }^{3}$ Department of Mechanical and Aerospace Engineering, Carleton University, Ottawa, Canada \\ ${ }^{4}$ Division of Engineering and Applied Science and Kavli Nanoscience Institute, California Institute of Technology, \\ Pasadena, California 91125, USA
}

(Received 9 February 2005; published 5 August 2005)

\begin{abstract}
Using a combination of statistical mechanics and finite-element interpolation, we develop a coarsegrained (CG) alternative to molecular dynamics (MD) for crystalline solids at constant temperature. The new approach is significantly more efficient than MD and generalizes earlier work on the quasicontinuum method. The method is validated by recovering equilibrium properties of single crystal $\mathrm{Ni}$ as a function of temperature. CG dynamical simulations of nanoindentation reveal a strong dependence on temperature of the critical stress to nucleate dislocations under the indenter.
\end{abstract}

DOI: 10.1103/PhysRevLett.95.060202

Many processes involving the physics and chemistry of materials result from microscopic interactions between the constituent atoms. As a result, molecular dynamics (MD) simulations pervade the literature of a variety of materialsrelated disciplines. However, large-scale atomistic simulations remain computationally demanding, resulting in the continued effort to seek alternatives which permit the examination of larger spatial domains or longer time scales.

An important step in this direction is a variety of multiscale methods which combine atomistic simulation with coarse-graining schemes (see [1] for a recent review). These methods exploit the fact that in many cases the critical dynamics may involve a relatively small subset of the entire set of atoms with the remainder of the atoms serving primarily to guarantee appropriate boundary conditions for the region of interest. One example is the quasicontinuum (QC) method, a zero-temperature energy minimization technique, which significantly reduces the total number of degrees of freedom that must be considered when simulating the deformation of crystalline solids $[2,3]$. In this method an approximation to the total potential energy is obtained by making use of finite-element constraints to remove atoms where the deformation field varies slowly on the scale of the lattice parameter. An attractive feature of this approach is its "seamlessness" in that the same underlying atomistic model is used in the energy calculations in both the coarse-grained (CG) and fully atomistic regions.

The aim of this Letter is to extend the QC method to treat the dynamics of systems at constant temperature. Our procedure is based on the concept of a potential of mean force (PMF), which was first introduced by Kirkwood in 1935 [4]. In principle, this approach enables the calculation of a variety of equilibrium and nonequilibrium properties of large systems using only a limited number of degrees of freedom. In practice, however, calculating the PMF directly from a molecular dynamics simulation is often com-
PACS numbers: 02.70.Ns, 02.70.Dh, 62.25.+g, 68.60.Dv

putationally demanding. This drawback can either make the PMF approach less efficient than the full atomistic calculation it is seeking to replace, or limits its use to linear coupling terms [5]. In this Letter we propose a method to substantially expedite the calculation of the PMF by making use of finite-element interpolation and the CauchyBorn rule [2]. As a result, the PMF can be recalculated at each time step, capturing in this manner the rich nonlinearity of the atomistic potential. We assess the validity of the method by comparing the temperature dependence of the lattice parameter of the CG system with that obtained from what we consider to be the "gold standard" for our method: a fully atomistic model described by the same interatomic potentials. In addition, we have developed the method to a degree that permits the study of general 2D deformation problems on 3D crystals with out-of-plane periodic boundary conditions. We demonstrate this capability by studying the temperature dependence of dislocation nucleation during nanoindentation.

Consider a system of $N$ atoms whose positions are denoted by $\{\mathbf{q}\}$. We assume the potential energy of the system $V(\{\mathbf{q}\})$ can be written as the sum of the energy $E_{i}(\{\mathbf{q}\})$ of each individual atom $i$. As shown in Fig. 1, we split the atom population ( $N$ atoms) between so-called representative atoms representative atoms, characterized by positions $\left\{\mathbf{q}^{r}\right\}$, which are the atoms that we will consider in our simulations and constrained atoms with coordinates $\left\{\mathbf{q}^{c}\right\}$. We define $N_{r}$ as the number of representative atoms and we will refer to this system as the CG system.

Following [4,6], we define the CG potential energy as the PMF for the constrained degrees of freedom,

$$
V_{\mathrm{CG}}\left(\left\{\mathbf{q}^{r}\right\}, \beta\right)=-\frac{1}{\beta} \ln \int e^{-\beta V\left(\left\{\mathbf{q}^{r}\right\},\left\{\mathbf{q}^{c}\right\}\right)} d\left\{\mathbf{q}^{c}\right\},
$$

where $\beta=1 / k_{B} T$. This particular choice guarantees that the ensemble-average of any observable $A$ (designated as $\langle A\rangle$ ) that depends only on the positions of the representative 


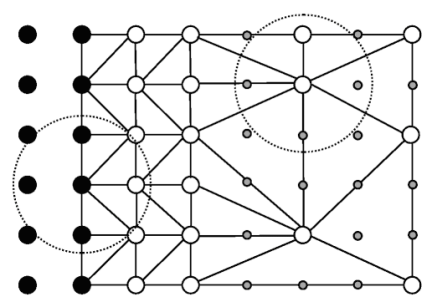

FIG. 1. We split the atom population between representative atoms (large circles on the figure) and constrained atoms (small gray circles). The average positions of the latter are estimated from the position of the former using finite-element interpolation. Among the representative atoms, we make a distinction between nonlocal atoms (black circles) whose energy only depends on representative atoms (as shown by the dashed circle whose radius equals the cutoff distance of the interatomic potential), and the local atoms (white circles) which interact with constrained atoms.

atoms $\left\{\mathbf{q}^{r}\right\}$ is equal to the ensemble average that would be found for this observable in a full atomistic and canonical system at equilibrium. That is,

$$
\left\langle A\left(\left\{\mathbf{q}^{r}\right\}\right)\right\rangle_{\mathrm{CG}} \equiv\left\langle A\left(\left\{\mathbf{q}^{r}\right\}\right)\right\rangle_{N_{r}, V, T}=\left\langle A\left(\left\{\mathbf{q}^{r}\right\}\right)\right\rangle_{\mathrm{N}, \mathrm{V}, \mathrm{T}} .
$$

Equation (2) provides a clear and reasonable objective for the CG methodology.

The Hamiltonian of the CG system is constructed as follows

$$
H_{\mathrm{CG}}\left(\left\{\mathbf{q}^{r}\right\},\left\{\mathbf{p}^{r}\right\}, \beta\right)=\sum_{i=1}^{N_{r}} \frac{\left(\mathbf{p}_{i}^{r}\right)^{2}}{2 m_{i}^{r}}+V_{\mathrm{CG}}\left(\left\{\mathbf{q}^{r}\right\}, \beta\right),
$$

where $\mathbf{p}_{i}^{r}=m_{i}^{r} \dot{\mathbf{q}}_{i}^{r}$ are the momenta of the representative atoms and $m_{i}^{r}$ are their effective masses. In most dynamical CG approaches the effective masses are computed using either a weighted mass matrix approach [5] or a lumpedmass approach [7]. Here we propose a more rigorous approach whereby the effective masses are obtained from two conditions: (1) that the total mass of the CG system equals that of the full-atom system, $\sum_{i} m_{i}^{r}=\mathrm{Nm}$; and (2) that both systems have the same momentum free energy $F_{p}$,

$$
F_{p}=-\frac{1}{\beta} \ln \prod_{i=1}^{N_{r}}\left(\frac{2 \pi m_{i}^{r}}{\beta h_{p}^{2}}\right)^{3 / 2}=-\frac{1}{\beta} \ln \left(\frac{2 \pi m}{\beta h_{p}^{2}}\right)^{3 N / 2},
$$

where $h_{p}$ is an arbitrary constant with dimensions of momentum [8]. These requirements are satisfied identically if $h_{p}=\sqrt{2 \pi m / \alpha \beta}$ and the effective masses are taken to be $m_{i}^{r}=\alpha^{n_{i}-1} m$, where $n_{i}$ is the number of atoms represented by representative atom $i$ (this is obtained from a Voronoi tesselation of the CG system [2]) and $\alpha$ is obtained from the solution of the equation $\sum_{i=1}^{N_{r}} \alpha^{n_{i}-1}=$ $N$. For the case of uniform coarse graining, where each representative atom represents $n=N / N_{r}$ atoms, the solution is $\alpha=\left(N / N_{r}\right)^{1 /(n-1)}$ and the effective masses are $m_{i}^{r}=\left(N / N^{r}\right) m=n m$. This corresponds to a simple lumped-mass approach. However, a lumped-mass approach is inappropriate for nonuniform coarse graining. For example, for a CG system comprised of 4 representative atoms representing, respectively, $n_{i}=1,2,3$, and 4 atoms, the effective masses are $m_{i}^{r}=\{1,1.661,2.758$, $4.581 \mathrm{~m}$. These are clearly different from the lumpedmass approach which simply gives $m_{i}^{r}=n_{i} m$.

In order to construct a dynamics for the representative atoms which allows for the simulation of systems in contact with a thermal reservoir, we adopt the Nosé-Poincaré thermostat $[9,10]$, though now applied to the set of representative atoms rather than all of the atoms. This approach defines a virtual CG microcanonical system, which ensures that ensemble averages of this system are exactly equal to averages of the original canonical system, thus preserving Eq. (2). Equations of motions can now be derived from the Hamiltonian of the microcanonical system. These equations can be integrated in turn using a time-reversible symplectic algorithm [11]. It should be noted that the resulting equations of motion for atoms in fully refined regions are identical to those of a full atomistic simulation.

The description given above is formally complete; however, we must still address the questions of how to efficiently implement these ideas, and how to expedite the calculation of the PMF $V_{\mathrm{CG}}\left(\left\{\mathbf{q}^{r}\right\}, \beta\right)$. Here we appeal to the QC formalism [2] to describe the configuration of the system. We first lay down a mesh between the representative atoms and decompose them into two sets as shown in Fig. 1. The nonlocal atoms (NL) are the atoms located in fully refined regions which do not interact with any constrained atoms. Their individual contribution $E_{i}\left(\left\{\mathbf{q}^{r}\right\}\right)$ to the CG potential can be calculated exactly based on the positions of the surrounding representative atoms as one would do in regular MD. On the other hand, local atoms interact with the constrained atoms in their vicinity. Taking advantage of the smoothness of the strain field in the CG regions, we use finite-element interpolation to express the thermally averaged positions of the constrained atoms as a function of the position of the representative atoms as $\left\langle\mathbf{q}_{j}^{c}\right\rangle=\Sigma_{i=1}^{N_{r}} S_{i j} \mathbf{q}_{i}^{r}$, where $S_{i j}$ are finite-element shape functions. In our implementation we use three-noded triangular elements with linear interpolation functions [12]. For computational efficiency, we also appeal to the local harmonic approximation suggested by LeSar [13,14], which has proven to be accurate for moderately strained crystals up to half the melting temperature. Using this approximation, the $\mathrm{CG}$ potential energy simplifies to

$$
V_{\mathrm{CG}}\left(\left\{\mathbf{q}^{r}\right\}, \beta\right)=\sum_{i=1}^{N} E_{i}\left(\left\{\mathbf{q}^{r}\right\}\right)+\sum_{i=1}^{N-N_{r}} \frac{1}{2 \beta} \ln \frac{\left\|\mathbf{D}_{i}\left(\left\{\mathbf{q}^{r}\right\}\right)\right\|}{(2 \pi / \beta)^{3}},
$$

where $E_{i}\left(\left\{\mathbf{q}^{r}\right\}\right)$ and $\left\|\mathbf{D}\left(\left\{\mathbf{q}^{r}\right\}\right)\right\|$ are, respectively, the energy and the determinant of the dynamical matrix of atom $i$. Finally we can take further advantage of the smoothness of the strain field in the CG regions and invoke the Cauchy- 
Born rule [2]. According to this approximation, all atoms in a single element in the CG region are assumed to all have an identical uniform environment characterized by the deformation gradient $\mathbf{F}_{e}$, which is calculated from the displacements of the three representative atoms $\left\{\mathbf{q}_{e}^{r}\right\}$ delimiting the element. The individual contributions of these atoms in the previous equation can then be replaced by a sum over elements:

$$
\begin{aligned}
V_{\mathrm{CG}}\left(\left\{\mathbf{q}^{r}\right\}, \beta\right)= & \sum_{i \in \mathrm{NL}} E_{i}\left(\left\{\mathbf{q}^{r}\right\}\right) \\
& +\sum_{e}\left[n_{e} E_{\mathrm{CB}}\left(\mathbf{F}_{e}\right)+\frac{n_{e}^{c}}{2 \beta} \ln \frac{\left\|\mathbf{D}_{\mathrm{CB}}\left(\mathbf{F}_{e}\right)\right\|}{(2 \pi / \beta)^{3}}\right],
\end{aligned}
$$

where NL is the set of nonlocal atoms, and $n_{e}$ and $n_{e}^{c}$ are, respectively, the total number of atoms and the number of constrained atoms in the element e. $E_{\mathrm{CB}}\left(\mathbf{F}_{e}\right)$ and $\left\|\mathbf{D}_{\mathrm{CB}}\left(\mathbf{F}_{e}\right)\right\|$ are, respectively, the potential energy and the determinant of the dynamical matrix of an atom embedded in an infinite perfect crystal subject to a uniform deformation gradient $\mathbf{F}_{e}$. The CG potential energy in Eq. (6) can be computed quickly and efficiently. It provides an adequate approximation for the PMF formally defined in Eq. (1) for temperatures up to about half the melting temperature. The essence of our model, then, is to represent the contributions of the thermal vibrations of the unrepresented atoms by treating them as harmonic oscillators, but where the entropy depends on the instantaneous local atomic configuration characterized by $\mathbf{F}_{e}$. For this reason the model is able to approximately capture phenomena in the $\mathrm{CG}$ region such as thermal expansion. This feature of the model is a consequence of the nonlinear finite deformation foundation of QC, which is lacking in most other approaches which assume small strain linear elasticity.

This model was implemented in the original version of the QC code [15]. The simulations are essentially 2D with periodic boundary conditions in the out-of-plane direction in order to mimic a 3D system. In Fig. 2 we compare the equilibrium lattice parameter of a defect-free single crystal obtained in the local regions with regular MD. The QC method gives similar results to those found using MD with a difference ranging from $0 \%$ at $0 \mathrm{~K}$ to $0.5 \%$ at $1000 \mathrm{~K}$, providing a quantitative assessment of the degree to which Eq. (2) is satisfied within our approximation of the PMF. The degradation of the results with increasing temperature is not surprising as anharmonic effects also increase [14]. The discrepancy between the two models is small relative to the standard deviation of atoms from their equilibrium positions (measured in the MD simulation) which range from $1.5 \%$ of the lattice parameter at $200 \mathrm{~K}$ to $5 \%$ at $1000 \mathrm{~K}$.

Next we turn to a more complex problem and investigate the temperature dependence of the threshold for dislocation nucleation during nanoindentation. This is an excellent application involving at once localized effects under

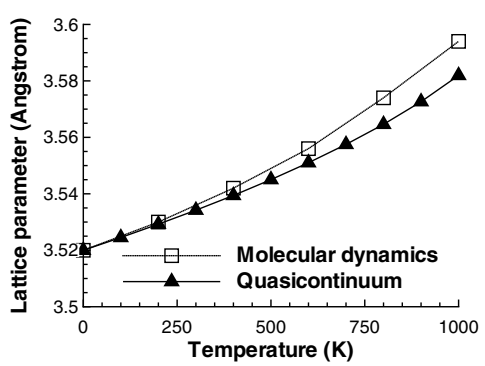

FIG. 2. Lattice parameter of nickel as a function of temperature using an embedded atom model (EAM) potential [22]. The melting temperature of this potential is $1478 \mathrm{~K}$ [23]. The QC calculation involved a cell of dimensions $200 \mathrm{~nm} \times 100 \mathrm{~nm}$ with a regular mesh containing 50 nodes. The lattice parameter was calculated as an average over the dimensions of the cell.

the indenter and long range effects due to elastic deformation fields. One of the features of nanoindentation experiments that make them especially appealing for multiscale simulations is that the experimental systems remain larger than the biggest cells that can be handled by MD creating a possible source of misinterpretation about the onset of dislocation activity [16]. Previous atomistic simulations have mostly been limited to zero temperature or small system sizes [17-21]. The use of the QC method is therefore compelling since it leads to a reduction in computational overhead that may permit in the future the direct simulation of experimental geometries at finite temperature. In the present work, a single crystal of $\mathrm{Ni}$ with dimensions $200 \mathrm{~nm} \times 100 \mathrm{~nm}$ was indented by a cylindrical indenter of radius $7 \mathrm{~nm}$ at temperatures ranging from $0 \mathrm{~K}$ to $400 \mathrm{~K}$ (see Fig. 3). The indentation direction coincides with the preferred slip direction [110], the hori-

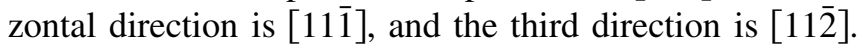
The mesh used in this simulation was fully refined beneath the indenter to allow dislocation nucleation. It contained only 3000 nodes (compared with 790000 atoms that would be required for full MD), thus providing considerable speed-up. The velocity of the indenter was chosen to be $5 \mathrm{~m} / \mathrm{s}$. Nanoindentation simulations were conducted after a $200 \mathrm{ps}$ equilibration time over a period of about $400 \mathrm{ps}$ with a time step of $1 \mathrm{fs}$.

The loading curves are presented in Fig. 4. Prior to dislocation nucleation, the curves follow an elastic loading path which is modified by the thermal dependence of the elastic coefficients. As is seen in Fig. 4, the onset of plasticity (i.e., dislocation nucleation) is affected by the temperature. Analysis shows that nucleation occurs under the indenter in the bulk in two stages as presented in Fig. 3. A more comprehensive study will be published elsewhere. We note that the zero-temperature results recover earlier results using the QC method in the absence of thermal effects [18].

In this Letter we have proposed a method for the dynamical simulation of crystalline solids at constant temperature. It captures both atomistic mechanisms and long 


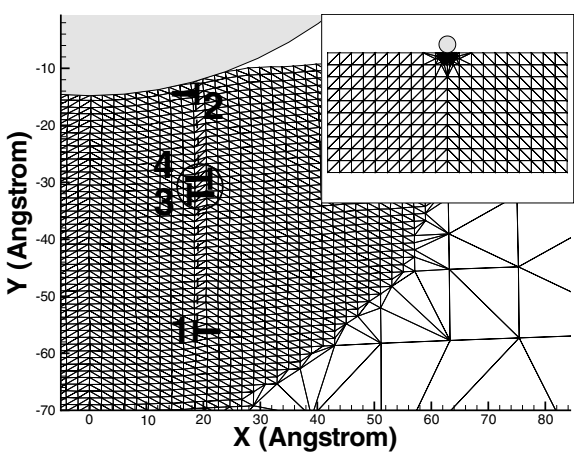

FIG. 3. Computational mesh at an indenter displacement of $1.5 \mathrm{~nm}$ at $100 \mathrm{~K}$, showing details of the nonlocal, fully atomistic region under the indenter as a dissociated edge dislocation nucleates. Elements between the representative atoms are drawn, rather than the representative atoms at the element vertices, to accentuate the deformation due to dislocation motion. Dislocation nucleation occurs in two stages. A first dipole of $\frac{1}{6} \times$ $\langle 112\rangle$ Shockley partial dislocations (SPD's) is nucleated under the surface (1 and 2). SPD 1 propagates into the bulk while SPD 2 reaches the surface, creating a stacking fault between them as shown by the dashed line. Later a second dipole of SPD's (3 and 4 ) is nucleated at the same location as the first dipole. SPD 3 propagates into the bulk to form a dissociated dislocation with SPD 1. SPD 4 moves to the surface, removing the stacking fault, and creating a step on the surface by combining with SPD 2. As temperature increases this mechanism occurs closer to the surface and on planes closer to the midline. The inset shows the entire initial mesh.

range effects without the computational cost of full atomistic simulations. We have shown that thermodynamic properties are in good agreement with conventional atomistic simulations. The ability of this method to investigate effects of temperature and defects in real structures has been demonstrated with the example of nanoindentation. Though these results provide an encouraging first step in the direction of MD simulations without all the atoms,

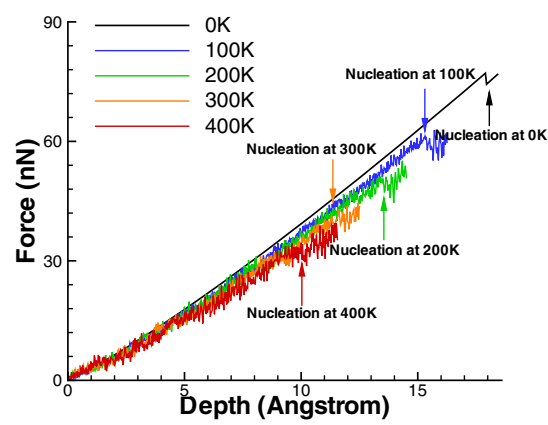

FIG. 4 (color online). Force vs indentation depth curve. The forces are averaged over a period of 0.5 ps to reduce thermal noise. The slope decreases with increasing temperature in accordance with the evolution of elastic coefficients. More interestingly, dislocation nucleation occurs at lower displacements with increasing temperature. there are a variety of interesting issues still to be explored including: how to carry out mesh adaption at finite temperatures, going beyond the local harmonic approximation used to compute the effective potential, and application to other problems of interest such as the temperature dependence of fracture.

We are grateful to Art Voter, Rob Rudd, Michael Ortiz, Jarek Knap, Bill Curtin, David Rodney, and Noam Bernstein for useful discussions. We gratefully acknowledge the support of CHSSI, CIMMS, MURI, and NSF NIRT. Part of this work was performed under the auspices of the US DOE by University of California, Lawrence Livermore National Laboratory under Contract No. W7405-Eng-48.

[1] G. Lu and E. Kaxiras, in Handbook of Theoretical and Computational Nanotechnology, edited by M. Rieth and W. Schommers (American Scientific Publisher, Stevenson Ranch, CA, 2005).

[2] E. B. Tadmor, M. Ortiz, and R. Phillips, Philos. Mag. A 73, 1529 (1996).

[3] V. B. Shenoy et al., J. Mech. Phys. Solids 47, 611 (1999).

[4] J. G. Kirkwood, J. Chem. Phys. 3, 300 (1935).

[5] R. E. Rudd and J. Q. Broughton, Phys. Rev. B 58, R5893 (1998).

[6] V. Shenoy, V. Shenoy, and R. Phillips, Mater. Res. Soc. Symp. Proc. 538, 465 (1999).

[7] E. Lidorikis et al., Phys. Rev. Lett. 87, 086104 (2001).

[8] K. Huang, Statistical Mechanics (Wiley, New York, 1987), 2nd ed..

[9] S. Nosé, Mol. Phys. 52, 255 (1984).

[10] S. D. Bond, B. J. Leimkuhler, and B. B. Laird, J. Comput. Phys. 151, 114 (1999).

[11] M. Tuckerman, B. J. Berne, and G. J. Martyna, J. Chem. Phys. 97, 1990 (1992).

[12] O.C. Zienkiewicz, and R. L. Taylor, The Finite Element Method (McGraw-Hill, New York, 1991), 4th ed..

[13] R. LeSar, R. Najafabadi, and D. J. Srolovitz, Phys. Rev. Lett. 63, 624 (1989).

[14] S. M. Foiles, Phys. Rev. B 49, 14930 (1994).

[15] The code can be downloaded at www.qcmethod.com.

[16] J. Knap and M. Ortiz, Phys. Rev. Lett. 90, 226102 (2003).

[17] C. L. Kelchner, S. J. Plimpton, and J. C. Hamilton, Phys. Rev. B 58, 11085 (1998).

[18] E. B. Tadmor, R. Miller, R. Phillips, and M. Ortiz, J. Mater. Res. 14, 2233 (1999).

[19] J. L. K. J. V. Vliet, T. Zhu, S. Yip, and S. Suresh, Nature (London) 418, 307 (2002).

[20] E. T. Lilleodden, J. A. Zimmerman, S. M. Foiles, and W. D. Nix, J. Mech. Phys. Solids 51, 901 (2003).

[21] J. Y. Hsieh, S.P. Ju, S.H. Li, and C.C. Hwang, Phys. Rev. B 70, 195424 (2004).

[22] J.E. Angelo, N. R. Moody, and M. I. Baskes, Model. Simul. Mater. Sci. Eng. 3, 289 (1995).

[23] F. J. Cherne, M. I. Baskes, and P. A. Deymier, Phys. Rev. B 65, 024209 (2002). 